\title{
Freundschaften, Symmetrien und Markierungen von Differenz
}

\author{
Aushandlungen und Konflikte in Peer-Beziehungen \\ aus Sicht von Kindern in Kindertageseinrichtungen
}

\author{
Stephanie Simon' ${ }^{1}$ Barbara Lochner ${ }^{2}$ und Werner Thole ${ }^{1}$ \\ ${ }^{1}$ Institut für Sozialwesen, Universität Kassel \\ ${ }^{2}$ Fachhochschule Erfurt
}

\begin{abstract}
Zusammenfassung: Der folgende Beitrag nimmt ausgehend von einem Forschungsvorhaben, welches Kinder als, Stakeholder ' in Kindertageseinrichtungen adressiert, in den Blick, wie Kinder im Kontext institutioneller Arrangements Peer- und Freundschaftsbeziehungen zum Thema machen. Es zeigt sich, dass die Formen der Thematisierung von verbindlichen „Wir“-Erzählungen bis zu komplexen, asymmetrischen Beziehungserfahrungen reichen.
\end{abstract}

Schlüsselwörter: Kindheit, institutionelle Arrangements, Freundschaften, Peers, Solidarität

Friendship, Symmetries, and Markings of Difference. Negotiations and Conflicts in Peer Relationships From the Perspective of Children in ECEC-Centers

\begin{abstract}
The article focuses on research about children as "stakeholders" in daycare centers, particularly on how children in this context address peers and friendship. We analyze how they see their peer context: Over the course of time, their narratives range from "we"-stories to the complexity of asymmetric relationships and orientations.
\end{abstract}

Keywords: childhood, institutional arrangements, early childhood education and care, friendship, peers, solidarity

Kindheiten spielten sich bis zum letzten Drittel des 20. Jahrhunderts vornehmlich auch auf der Straße ab. In der lesenswerten Studie „Kinder unter sich“ (Behnken \& du Bois-Reymond, 1991) wird nachdrücklich daran erinnert, dass sich Kindergruppen draußen, im wohnortnahen Quartier, keineswegs über gesellschaftliche Institutionen wie Schulen, Vereine oder Kindertageseinrichten konstituierten. Insbesondere jüngere Kinder gestalteten, wird den Berichten gefolgt, ab den 1970er Jahren eine kindliche „Wirklichkeit aus zweiter Hand“ (Bauer \& Hengst, 1980), eine „verhäuslichte“ Kindheit, auch weil „frei bewegen in selbst eroberten Geländen“ (Berg, 1991, S.23) als gefährlich galt. Zunehmend intensiver erleb(t)en Kinder Kindheiten in „,verinselten“ Refugien und organisierten Arrangements wie Sportvereinen, Kunst- und Musikschulen oder in parzellierten, eingezäunten Spielflächen.

Entwickelten sich vormals Kinderkulturen selbstorganisiert in und über städtische und ländliche Sozialräume
(Zinnecker, 2001; Muchow \& Muchow, 1935), so heute vornehmlich in institutionellen Angeboten des Bildungsund Sozialsystems. Wie für viele ältere Kinder und Jugendliche heute Schule der Ort ist, wo soziale Kontakte entstehen, sind für jüngere Kinder Kindertageseinrichtungen zentrale „Inseln“ des Aufwachsens. Hier bilden sich erste eigenständige Peer-Kulturen (Corsaro, 2009), über die Kinder soziale Netzwerke und Gemeinschaft (Göbel, 2018) herstellen, reproduzieren oder eigensinnig transformieren. Kindertageseinrichtungen können die Selbstorganisation von Kindern in Gruppen ermöglichen und rahmen (Brandes \& Schneider-Andrich, 2017), insbesondere wenn diese außerhalb der Institutionen wenig Kontakt zu anderen Peers haben (Ahnert, 2003).

Als Peers ${ }^{1}$ werden Gruppen bezeichnet, die über Alter, Entwicklungsstand oder einen ,gleiche[n] Status gegenüber [einer] Institution" (Siebholz \& Winter, 2014, S.397) bzw. eine "Gleichheit der Stellung im Verhältnis zueinander" (Krappmann, 2010, S. 200) gekennzeichnet sind. An-

Vom Peer-Konzept wird in psychologischer Perspektive das Freundschafts-Konzept differenziert (Selman, 1980). 
dere Definitionen heben interaktiv hergestellte Praktiken und Orientierungen hervor (Brake \& Büchner, 2013). Bildungspolitisch wird die Relevanz von Peers in Kindertageseinrichtungen bislang selten auf die Tagesordnung gesetzt, obwohl etwa während der flächendeckenden Schließungen dieser Orte aufgrund der Corona-Pandemie erste empirische Befunde zu den Auswirkungen der Maßnahmen hervorheben, dass es besonders die sozialen und emotionalen Interaktionen zu Peers sind, die den Kindern fehlen (u.a. Lochner, 2020, S.3).

Ausgehend von theoretischen und empirischen Vergewisserungen zum Thema Freundschaften und Peer-Beziehungen (1) werden im folgendem Beitrag auf Basis von ersten Befunden aus dem Projekt „Kinder als ,Stakeholder ' in Kindertageseinrichtungen“" (2) die Relevanzen von Peer-Beziehungen und Freundschaften aus Sicht der Kinder betrachtet (3) sowie in ihrer Relevanz für die pädagogische Praxis beleuchtet (4).

\section{Peer-Beziehungen - Theoretische und empirische Vergewisserungen}

Es kann als Allgemeinwissen gelten, dass Kinder untereinander anders interagieren als mit Erwachsenen (Piaget, 1976), da Kinder unter sich stärker zur Perspektivübernahme herausgefordert seien (Edelstein \& Keller, 1982), während Kinder gegenüber Erwachsenen dazu neigten, ,aus Liebe, Anerkennung oder auch Furcht [...] zu folgen" (Krappmann, 2010, S.187). Reflexivität und Wissen über die eigene Positioniertheit sowie Kooperation würden in Peer-Interaktionen angeregt (Youniss, 1982), da diese frei von Hierarchie seien.

Dieser entwicklungspsychologische Befund grundiert weitere Studien zur Relevanz von Peer-Kontakten. So werden Peer-Interaktionen als Entwicklungsressource (Ahnert, 2003) angesehen - z.B. für wechselseitige Bezugnahmen und Ko-Konstruktionen (Youniss, 1994), Mentalisierungsprozesse (Fonagy et al., 2019) und die Ausbildung von $\mathrm{Zu}$ - und Zusammengehörigkeitsgefühlen (Brandes \& Schneider-Andrich, 2017). Die Erfüllung des Bedürfnisses sozialer Eingebundenheit wird lerntheoretisch als Grundvoraussetzung für die Aneignung von Welt, Wissen und Sprache gesehen (Deci \& Ryan, 1993).

Bereits diese Hinweise verdeutlichen, dass kindliche Entwicklung nicht nur individuell verläuft, sondern immer auch durch (aus)handlungsorientierte Prinzipien bestimmt werden, welche die Sozialwelt von Kindern und damit auch die Struktur ihrer Lebenswelt ko-konstruieren (Youniss, 1982). Kindertageseinrichtungen können diesbezüglich als institutioneller Rahmen verstanden werden, in denen sich Praktiken der Aushandlung innerhalb der Kinderwelt intergenerational aufschichten, reproduzieren und transformieren (Krappmann, 1996).

Kinder-Freundschaften verändern sich mit zunehmenden Alter (Wehner, 2016). Unter-zweijährige Kinder handeln nonverbal Gemeinsamkeiten, Spiele und Auseinandersetzungen mit Peers aus (Viernickel, 2000). Für vier- bis siebenjährige Kinder wird eine Asymmetrie in Freundschaftsbeziehungen proklamiert (Selman, 1980), die sich dadurch auszeichne, dass Kinder Forderungen ans Gegenüber stellen, bis sich mit ansteigendem Alter eine Symmetrie der Freundschaftsdyade herausstelle. Studien zu Peer-Beziehungen weisen zudem auf das Bedürfnis von Kindern nach Eingebundenheit und Zugehörigkeit hin - insbesondere auch im Ablöseprozess der Eltern (Hover-Reisner et al., 2018).

Die sozialwissenschaftliche Kindheitsforschung nimmt Kinder als Akteur_innen in den Blick und versucht auf diese Weise, kindliche Lebenswelten weniger adultistisch zu deuten (vgl. u.a. Eßer et al., 2016). Die so generierten Befunde - Entwicklung von Reziprozität und gemeinsamer Spielkultur, aber auch die Thematisierung von Konflikten (Nentwig-Gesemann et al., 2020) - legen nahe, davon auszugehen, dass auch in Peer-Beziehungen komplexe Aushandlungsprozesse in Machtverhältnisse eingewoben und somit nie frei von Hierarchiesierungen und darüber produzierte Ungleichheiten sind (Betz \& de Moll, 2019; Machold, 2015). Vor dem Hintergrund ließe sich annehmen, dass Kinder sich und andere entlang anderer Differenzkategorien positionieren, sich zueinander ins Verhältnis oder auch in Konkurrenz setzen.

\section{Kindertageseinrichtungen aus Sicht von Kindern - zum Forschungsvorhaben}

In diesem Beitrag wird auf Material des Forschungsprojektes „Kinder als ,Stakeholder' in Kindertageseinrichtungen" (KiSte) (Laufzeit 2018-2021)² zurückgegriffen. Dort wird davon ausgegangen, dass Kinder als Adressat_innen, Nutzer_innen und Transformator_innen pädagogischer Arrangements Interessen verfolgen, die unterschiedlich wahrgenommen werden und für die nicht 
immer Formate der Artikulation zur Verfügung stehen (Witton et al., 2020; Lochner et al., 2020). Im Forschungsprozess werden Kinder als zentrale Akteur_innen in Kindertageseinrichtungen adressiert, um ihnen die Möglichkeit zu geben, ihre Sichtweisen zu kommunizieren. So wird empirisch fundiertes Wissen bezüglich der differenten Deutungen von Kindertageseinrichtungen durch Kinder generiert.

Kinder werden animiert, mit Videokameras den Forscher_innen die für sie relevanten Orte, Akteur_innen, Artefakte, die Infrastruktur und pädagogische Praktiken ihrer Institution zu zeigen und zu erläutern. Die von den Kindern erstellten Videografien werden seitens der Forscher_innen durch dialogische Interviews angereichert. Mittels sequentieller Rekonstruktionen werden die Transkripte der Gespräche unter Beachtung des Kodierparadigma der Grounded Theory und der Rekonstruktionsschritte der dokumentarischen Methode analysiert (Bohnsack 2014).

Forschungsmethodologisch wird davon ausgegangen, dass Kinder in der Lage sind, offene Erzählaufforderungen aufzunehmen und eigensinnig zu transformieren. So konnten bislang aus 14 Einrichtungen in 105 Videospaziergängen visuelles Bildmaterial sowie auditives Gesprächsmaterial erhoben werden. Obschon die Kinder über den Erzählstimulus nachdrücklich und offen motiviert werden, das zu zeigen, was ihnen wichtig ist und was ihnen besonders gefällt oder nicht, zeigen die Materialsichtungen, dass Peers von den Kindern fast durchgehend präsentiert und thematisiert werden. Peer-Interaktionen und -Beziehungen werden von den Kindern in den Gesprächen eng mit ihren Perspektiven auf das institutionelle Arrangement verwoben. ${ }^{3}$

In diesem Beitrag erfolgt eine Konzentration auf die Sprechakte in den Videografien. Durch diese Fokussierung erfahren Aufnahmen, in denen Kinder weniger erzählend und eher handelnd agieren, eine geringere Aufmerksamkeit. Ob aus einer habitus- und ungleichheitssensiblen Perspektive damit eine sprech- gegenüber einer handlungsorientierten Perspektive eine bedeutsame Priorität erfährt, ist über weitere Analysen zu prüfen. Den nachfolgenden Ausführungen liegen drei, als zentral identifizierte Fälle zugrunde. Sicherlich kann so das Tableau von möglichen Thematisierungsweisen von Freundschaft in Kindertageseinrichtungen nicht umfänglich dokumentiert werden. Aufgrund des vorliegenden Untersuchungsmaterials kann jedoch davon ausgegangen werden, dass sich in der Auswahl nicht lediglich subjektive, sondern strukturelle, gesellschaftlich grun- dierte Thematisierungsformen von Peer-Beziehungen zeigen.

\section{Empirische Erkundungen}

Die Befunde zu kindlichen Freundschaften regen Fragen an, ob und wie Peers und Freundschaftsbeziehungen von Kindern thematisiert werden. Anhand der transkribierten Videografien von Kindern wird aufzuschlüsseln versucht, welche Konzepte von Peer-Beziehungen und Freundschaften dabei artikuliert werden.

\section{„Mit jedem spiele ich gerne, weil das alles meine Freunde sind" - Deskriptive Annäherung}

Bereits eine erste Annäherung ans Material eröffnet mit Blick auf die Thematisierung von Peer-Beziehungen und Freundschaften ein komplexes Bild. In den bildlichen Darstellungen werden über die Form, wie Peers präsentiert werden, diese schon als bedeutsam präsentiert. Auf verbaler Ebene werden Peer-Beziehungen und Freundschaften sowohl selbstläufig thematisiert als auch auf Nachfrage. In den Erzählungen kommen Konflikte um oder die gemeinsame Nutzung von Spielgeräten und -materialien ebenso zur Sprache wie das Thema Freundschaft. Dabei werden Abstufungen wie „Freunde“ und „richtige Freundin“ vorgenommen. Neben konkreten Freund_innen, die namentlich eingeführt werden, werden Peers als Kindergruppen (,wir“) thematisiert. Das Sprechen darüber reicht von detaillierten, dichten, emotionalen Beschreibungen bis zu unspezifischen Erzählungen.

Auf bildlicher Ebene wie auch in den Narrationen lassen sich performative Herstellungspraktiken von Zugehörigkeit und Abgrenzung zu Peers identifizieren. Darüber hinaus verdeutlicht der kursorische Blick auf kindliche Thematisierungen, dass die gemeinsame Beschäftigung oder die geteilte Aufmerksamkeit für eine Sache von Bedeutung für das Ausweisen von Zugehörigkeit sein kann. Eine zentrale Form, um „Peer-Gemeinschaft“ auszudrücken, wird zudem darüber angedeutet, dass Spielpräferenzen als kollektive Ideen präsentiert werden. Das Sprechen im „Wir“ verweist auf eine gegenseitige Bezugnahme und beinhaltet, sich als Teil eines Kollektivs zu verstehen, sowie darüber hinaus sich in der Lage zu fühlen, für dieses sprechen zu können (Lochner, 2017, S.150). Diese knappen Ein-

\footnotetext{
Anhand des von den Kindern erhobenen Videomaterials ist die Repräsentation von Peer-Beziehungen im Bild eine weitere Analyseebene, die insbesondere in Kombination mit den Äußerungen der Kinder aufschlussreich ist. Für den hier gewählten Fokus auf die Thematisierung von Aushandlungen und Differenzen in Freundschaftsbeziehung bot sich die Konzentration auf Verbaldaten an.
} 
blicke ins empirische Material sollen nachfolgend anhand von Sequenzen aus drei Gesprächen vertiefend aufgeschlüsselt werden.

\section{Freundschaften und Peer-Interaktionen als komplexe Konstrukte}

\section{Kooperation und Asymmetrie: „, no nie ham wir etwas für meine Mama gemacht“"}

Maya (sechs Jahre) thematisiert auf die Frage nach ihren Freund_innen insbesondere den Kontakt zu ihrer ,allerbesten Freundin" Aiko. Sie illustriert das Konfliktpotenzial der Beziehung über Situationen, in der auch Aikos jüngere Schwester Frieda beteiligt ist:

\section{M: [...] Aiko un Frieda streitn manchma ganz oft mit mir mh weil (.) immer machn wir nur Sachn für Aiko un Frieda mh Mama weil sie ständig Geburtstag hat (.) un no nie ha ham wir ge etwas für meine Mama gemacht //l: ok// zusammen (Maya, Z.173-176)}

Das gemeinsame Herstellen von „Sachen für [Mama]“ erzeugt zwischen Maya und Aiko einen, die Beziehung der beiden Freundinnen ins Wanken bringenden „Streit“. Der Erzählung folgend werden in Situationen des gemeinsamen Produzierens immer nur Dinge für die Mutter von Aiko und Frieda hergestellt, da deren „Mama ständig Geburtstag hat". Maya konstatiert, dass sie „no[ch] nie etwas für meine Mama gemacht“ haben, „zusammen“. Obwohl von ihr weder der Konflikt in der kooperativen Praxis thematisiert noch das Argument infrage gestellt wird, wird die Asymmetrie und Ungerechtigkeit, die Maya in den entsprechenden Situationen meint zu verspüren, deutlich. Sie artikuliert nicht, sich nicht für Aikos und Friedas Mutter mit engagieren zu wollen, sondern moniert die fehlende Wechselseitigkeit des Freundschaftsdienstes. Es deutet sich hier ein machtvolles Freundschaftsverhältnis an, bei dem Maya sich als diejenige moduliert, die unterstützt, ohne in gleicher Weise Unterstützung zu erfahren. An einer späteren Stelle legen Mayas Äußerungen nahe, dass diese Streitigkeiten zwar dazu dienen, Begründungen und reziproke Handlungsweisen einzufordern, die Stabilität der Beziehung dadurch aber nicht gefährdet ist, denn obwohl „wir uns streiten, sind wir immer noch beste Freunde“.

\section{Reziprozität und wechselseitige Wahrnehmung: "am meisten lach ich und Messi und Lukas"}

Rio (sechs Jahre) beschreibt auf Nachfrage, wie er im Garten mit „[s]einen Freunden wie Messi und Lukas und Rafael und Jan“ (Z.49) spielt. Die Darstellung seiner Freunde als Subjekte entfaltet im Verlauf des Interviews eine hohe Relevanz. In Äußerungen zur Einrichtung greift
Rio detailliert auf Erfahrungen und Interaktionen mit Peers, insbesondere mit jenen, die er als Freunde markiert, zurück. So auch in folgender Sequenz einer Erzählung zum Mittagessen:

I: Und was ist das für ein Quatsch den ihr drin macht?

R: Quatsch machen wir so welche äh Sachen und da lachen manche Kinder und dann manche lachen auch mal nicht aber am meisten lach ich und Messi und Lukas und ich lachen und dann und dann lacht Messi manchmal nicht und dann lach ich wenn der Lukas lustige Sachen macht und dann mach ich und wenn ich zu Lukas zu Messi lachen dann lacht nur Lukas und wenn Messi noch lustige Sachen macht dann lach ich und Lukas (Rio, Z. 83 -103).

Rio beschreibt differenziert die wechselseitige Affizierung der Kinder. Die Aktivitäten des "Quatsch machen“ erzeugen unterschiedliche Reaktionen. Laut Rio ist der "Quatsch" nicht für die gesamte, abstrakt bleibende PeerGroup lustig. Zudem beschreibt er die unterschiedliche Involviertheit seiner Freunde in die Aktivität: Erstens lachen sie in unterschiedlicher Intensität, zweitens sind nicht alle Animateure des gemeinsamen Quatschmachens und drittens wird in seiner Äußerung die ko-konstruktive, reziproke Entwicklung der Handlung relevant gemacht, die Rio beobachtend als wenn-dann-Folge formuliert und differenziert. Rio beobachtet sich und seine unterschiedlichen Freundschaftsbeziehungen kleinteilig. Er beschreibt, wie in alltäglichen Situationen Zugehörigkeit, emotionale Affizierung, aber auch Abgrenzung zu anderen - den NichtBenannten - performativ hergestellt werden. Nicht über die Peer-Group an sich, sondern über die gemeinsamen Aktivitäten mit benennbaren Anderen stellt er seinen Bezug auf das institutionelle Arrangement her.

\section{Aktiv sein mit anderen: „wenn wir dann ma nich Freunde findn das is dann immer blöde"}

Bei Charlotte (sechs Jahre) steht das gemeinsame Aktivsein mit anderen im Mittelpunkt:

C: aber sonst find ich eigntlich die Freunde immer gu::.: un ich findes wichtig dass man dass ich un meine Freunde immer also die könn ja auch so zu sagn manchma sind wa nich so gut gut zusammn dass ich dass ich jetz ma sage ich möchte ma was anderes mit jemand machn aber eigentlich (unv. 1 Sek.) aber wenn wenn wir dann ma nich Freunde findn das is dann immer blöde weil dann hat man niemandn zum spieln (.) (Charlotte, Z. 80 - 97)

Vor diesem abwägenden Fazit zeichnet Charlotte jedoch ein komplexes, dynamisches Bild von Gruppenpro- 
zessen, denen sich Kinder ausgesetzt fühlen (können), wenn Aktivitäts- und Beziehungswünsche nicht übereinstimmen:

C: ja und vorallem die die Jungs gebn auch so schnell auf dass sie sagn ne un wir Mädchen das die wolln halt auch mit den Jungs spieln (schniefen) un dann un das is halt $\mathrm{n}$ bisschn blöd und dann (schniefen) müssn mir halt auch aufgebn obwohl wir das ma grade wolln (Charlotte, Z. $73-76)$.

In Charlottes Erzählung wird den „Jungs“ wie selbstverständlich mehr situative Gestaltungsmacht zugewiesen. Dies kann als Hinweis gelesen werden, dass sie nicht nur eine sich wiederholende Differenz markiert, sondern diese als latent präsent, auf geschlechterförmigen Zuschreibungen und Praktiken basierend, reflektiert. Über die Äußerung ,is halt n bisschn blöd" artikuliert Charlotte ihre Missbilligung des wahrgenommenen Ungleichgewichtes in ihrem Freundschaftsnetzwerk, da so auch deswegen Aktivitäten abgebrochen werden müssen, weil nicht alle Kinder in gleicher Weise an diesen interessiert sind. Zugleich betont sie aber in ihrem Resümee, dass sie „aber sonst“ „die Freunde gut" findet. Sie relativiert damit die von ihr als problematisch markierte Spiel-Situation als Ausnahme und elaboriert abschließend, was ihr an Freundschaften wichtig ist: Sie findet einen reziproken, offenen Austausch zu eigenen Bedürfnissen wichtig, der Unstimmigkeiten überwindet - ,manchma sind wa nicht so gut zusamm". Sie erkennt aber auch die Gefahr einer offenen Kommunikation, nämlich am Ende mit der gewählten Aktivität alleine zu sein.

Charlotte scheint den Erhalt von Peer-Beziehungen über ihre individuellen Spielpräferenzen zu stellen. Die Kindertageseinrichtung adressiert sie in ihren Äußerungen als Ort, an dem Freundschaften ausgehandelt und Aktivitäten in ihrer Bedeutung mit anderen Kindern kollektiv entwickelt werden können. Gleichzeitig deutet sich in ihrer Erzählung über ihre Feststellung, „wir Mädchen, die wollen halt", eine genderspezifische Aufmerksamkeit gegenüber den nicht unabhängig von gesellschaftlichen Machtstrukturen gestaltenden Spiel- und Aktivitätswahldynamiken an.

\section{Vergleichende Betrachtung}

In den drei Sequenzen werden Freundschaften als Beziehungen attribuiert, die relativ stabil sind, Aushandlungen und Konflikte aushalten, in denen Reziprozität erwartet und das Gegenüber als Subjekt adressiert und wahrgenommen wird. Im Mittelpunkt steht ein gemeinsames, beziehungsorientiertes Handeln. Diese Befunde korres- pondieren mit der Erkenntnis, wonach symmetrische Reziprozitätserwartungen sozialisationstheoretisch der Entwicklung von Kooperation dienen, über die sich wiederum Solidarität ausbilden kann (Youniss, 1982, S. 82ff.).

Während bei Maya und Rio eine Differenz im Binnenund Außenverhältnis des Beziehungsgefüges artikuliert wird, verweist Charlottes Äußerung auf wirkmächtige Geschlechterkonstruktionen in den Praktiken der Kindergruppe und damit auf eine strukturelle Differenz. Dies bietet Anlass für ungleichheitstheoretische Überlegungen (vgl. Machold, 2015). Ausgehend von der Annahme einer subjektbildenden Wirkung solcher Aushandlungen, empfiehlt diese Sequenz, der Machtförmigkeit der von Charlotte zum Ausdruck gebrachten, auf Geschlecht bezogenen Praktiken in der Beziehungsgestaltung in Kindertagesstätten nachzugehen (vgl. Kubandt, 2016). Deutlich wird, dass sie sich nicht durchgängig der Mädchengruppe zuordnet, da sie auch distanziert von „den Mädchen“ spricht. Gleichzeitig bringt sie sich in der von ihr beschriebenen Praktik des Folgens der Mädchengruppe, die wiederum den Spielpräferenzen der Jungengruppe folgt, als ein Mädchen hervor, das nicht nur Geschlecht als Kategorie subjektiv verinnerlicht hat, sondern auch das damit zusammenhängende gesellschaftliche Machtungleichgewicht.

\section{Resümee und Implikationen für die pädagogische Praxis}

Die Analysen zeigen, dass institutionelle Arrangements wie Kindertageseinrichtungen Arenen darstellen, die Kinder „bespielen“, um Freundschaftsnetzwerke herzustellen und $\mathrm{zu}$ gestalten. Bestätigt werden damit vorliegende Befunde, dass Gemeinschaft, Spiel und Konflikt zentrale Themen für Kinder sind (Viernickel, 2000; NentwigGesemann et al., 2020). Die von Kindern in dem Vorhaben „Kinder als ,Stakeholder" in Kindertageseinrichtungen“ artikulierten und über Rekonstruktionen aufgeschlossenen Sichtweisen zeigen darüber hinaus erstens, dass Kinder ihre im institutionellen Kontext hergestellten Freundschaftsbeziehungen thematisieren können, Ambivalenzen und schwierige Konfigurationen dieser zweitens identifizieren und reflektieren können sowie drittens zwischen dem Erhalt von Freundschaften und konflikthaften Disharmonien abwägen. Dabei finden viertens die Erfahrung von sozialen Ungleichheiten ebenso Eingang in die Thematisierung von gemeinsamen Spielpraktiken wie asymmetrische Beziehungs-Erfahrungen, die zwischen individuellen und kollektiven Bedürfnissen austariert werden müssen. Wenn zudem davon ausgegangen wird, dass Kin- 
der in Interaktionen auch auf Solidarität ${ }^{4}$ abzielen, sich gegenseitig helfen und unterstützen (Tomasello, 2011) und Konkurrenz etwas ist, was erst im Laufe des Aufwachsens über Bildungsinstitutionen - und deren Fokus auf individueller Förderung - erworben wird, machen die hier vorliegenden Befunde deutlich, wie Kinder eigene Interessen zur Aufrechterhaltung von Freundschaftsbeziehungen eher zurückstecken, obschon sie darin enthaltene Differenzen reflektieren und als ungerecht thematisieren.

Der methodische Zugang des Forschungsvorhabens ermöglicht, den Sicht- und Kommunikationsweisen von Kindern ohne Handlungsdruck nachzuspüren. Deutlich wird dabei aber auch, wie wichtig es für die pädagogische Praxis ist, sich beobachtend und handlungsentlastet den Perspektiven der Kinder zu nähern, um ihnen Raum zu geben und sie nicht normativ zu überformen. Die Beobachtungen könnten für Pädagog_innen als Empfehlung verstanden werden, mit Kindern diesbezüglich ins Gespräch zu kommen, dabei sensibel auf von ihnen wahrgenommene Asymmetrien, Ungleichheiten und ggf. auch Machtverhältnisse einzugehen, um Kinder darin zu unterstützen, stabile Beziehungen zu entwickeln und sich selbst in diesen solidarisch zu positionieren.

\section{Literatur}

Ahnert, L. (2003). Die Bedeutung von Peers für die frühe Sozialentwicklung des Kindes. In H. Keller (Hrsg.), Handbuch der Kleinkindforschung (S.493 - 528). Bern: Huber.

Bauer, K. W. \& Hengst, H. (1980). Wirklichkeit aus zweiter Hand. Reinbek b. Hamburg: Rowohlt.

Behnken, I. \& Bois-Reymond, M. (1991). Kinder unter sich. In C. Berg (Hrsg.), Kinderwelten (S.103-130). Frankfurt a.M.: Suhrkamp.

Berg, C. (1991). Kinderleben in der Industriekultur. In C. Berg (Hrsg.), Kinderwelten (S.15 - 39). Frankfurt a. M.: Suhrkamp.

Betz, T. \& de Moll, F. (2019). Ungleichheitsbezogene Bildungsforschung - Kinder als Reproduzenten von Bildungsungleichheit. Zeitschrift für Soziologie der Erziehung und Sozialisation, 39, $225-228$.

Bohnsack, R. (2014). Rekonstruktive Sozialforschung. Einführung in qualitative Forschung (9. Aufl.). Opladen: Barbara Budrich

Brake, A. \& Büchner, P. (2013). Stichwort: Familie, Peers und (informelle) Bildung im Kindes- und Jugendalter. Zeitschrift für Erziehungswissenschaft, 16, $481-502$.

Brandes, H. \& Schneider-Andrich, P. (2017). Die Bedeutung der Gleichaltrigen in Kindertageseinrichtungen. Frühe Kindheit, 2, $22-29$.

Corsaro, W. A. (2009). Peer Culture. In J. Qvortrup, W. A. Corsaro \& M.-S. Honig (Eds.), The Palgrave Handbook of Childhood Studies (S. 62 - 77). Basingstoke: Palgrave Macmillan.

Deci, E. L. \& Ryan, R. M. (1993). Die Selbstbestimmungstheorie der Motivation und ihre Bedeutung für die Pädagogik. Zeitschrift für Pädagogik, 39, $223-238$.
Edelstein, W. \& Keller, M. (Hrsg.). (1982). Perspektivität und Interpretation. Beiträge zur Entwicklung des sozialen Verstehens. Frankfurt a. M.: Suhrkamp.

Eßer, F., Baader, M. S., Betz, T. \& Hungerland, B. (Eds.). (2016) Reconceptualising Agency and Childhood. London: Routledge.

Fonagy, P., Gergely, G., Jurist, E. L. \& Target, M. (2019). Affektregulierung, Mentalisierung und die Entwicklung des Selbst (7. Aufl.). Stuttgart: Klett-Cotta

Göbel, S. (2018). Konstruktion von Gemeinschaft im frühpädagogischen Alltag. In I. Kaul, D. Schmidt \& W. Thole (Hrsg.), Kinder und Kindheiten. Studien zur Empirie der Kindheit. Unsicherheiten, Herausforderungen und Zumutungen (S.55-74). Wiesbaden: Springer VS.

Hover-Reisner, N., Fürstaller, M., Datler, W. \& Datler, M. (2018). Institutionalisierung der frühen Kindheit als Prozess der Erweiterung des sozialen Umfelds: Peers und ihre Bedeutung für frühe Bildungs- und Entwicklungsprozesse im Krippen- und Kindergartenkontext. In B. Bloch et al. (Hrsg.), Kinder und Kindheiten. Frühpädagogische Perspektiven (S.281 - 293). Weinheim: Beltz Juventa.

Jaeggi, R. \& Celikates, R. (2017). Sozialphilosophie. Eine Einführung. München: C. H. Beck.

Krappmann, L. (2010). Prozesse der kindlichen Persönlichkeitsentwicklung im Kontext von Gleichaltrigenbeziehungen. In M. Harring et al. (Hrsg.), Freundschaften, Cliquen und Jugendkulturen (S.187-222). Wiesbaden: VS.

Krappmann, L. (1996). Streit, Freundschaft und Aushandlungen unter Kindern. In M.-S. Honig, H. R. Leu \& U. Nissen (Hrsg.), Kinder und Kindheit. Soziokulturelle Muster - sozialisationstheoretische Perspektiven (S.99-116). Weinheim: Juventa.

Kubandt, M. (2016). Geschlechterdifferenzierungen in der Kindertageseinrichtung. Eine qualitativ-rekonstruktive Studie. Opladen: Barbara Budrich.

Lochner, B. (2017). Teamarbeit in Kindertageseinrichtungen. Wiesbaden: Springer VS.

Lochner, B. (2020). Familien in Zeiten von Corona - Wohlbefinden der Kinder, Herausforderungen des Homeschooling \& Unterstützungsbedarfe der Eltern. Erste Befunde. Verfügbar unter: https://www.dksbthueringen.de/fileadmin/user_upload/pdf/ Praesentationen/20-04-25_Befr.Familien-1.Befunde.pdf

Lochner, B., Witton, T. \& Kim, M. (2020). Adressat*innen als Stakeholder pädagogischer Organisationen. In P. Cloos, B. Lochner, H. Schoneville (Hrsg.), Soziale Arbeit als Projekt. Konturierungen von Disziplin und Profession (S.323 - 336). Wiesbaden: Springer VS.

Machold, C. (2015). Kinder und Differenz. Eine ethnografische Studie im elementarpädagogischen Kontext. Wiesbaden: Springer VS.

Muchow, M. \& Muchow, H. H. (1935). Der Lebensraum des Großstadtkindes. Hamburg: Martin Riegel.

Nentwig-Gesemann, I., Bakels, E., Walther, B. \& Munk, L.-M. (2020). Kinder als Akteure in Qualitätsentwicklung und Forschung. Eine rekonstruktive Studie zu KiTa-Qualität aus der Perspektive von Kindern. Gütersloh: Bertelsmann.

Piaget, J. (1976). Die Äqulibration der kognitiven Strukturen. Stuttgart: Klett-Cotta.

Selman, R. L. (1980). The growth of interpersonal understanding. Developmental and clinical Analyses. New York, NY: Academic Press.

Siebholz, S. \& Winter, D. (2014). Peers in der frühen Kindheit. In R. Braches-Chyrek, C. Röhner, H. Sünker \& M. Hopf (Hrsg.), Handbuch Frühe Kindheit (S.397 - 407). Opladen: Barbara Budrich.

4 Solidarität kann auch gegenüber Fremden ausgedrückt werden, welche nicht, in der direkten Wechselseitigkeit von Leistung und Gegenleistung [...], sondern über die Identifikation mit gemeinsamen Zielen“" (Jaeggi \& Celikates, 2017, S.39) und über gemeinsame Praktiken herstellt wird. 
Tomasello, M. (2011). Warum wir kooperieren. Frankfurt a.M.: Suhrkamp.

Viernickel, S. (2000). Spiel, Streit, Gemeinsamkeit. Einblicke in die soziale Kinderwelt der unter Zweijährigen. Landau: Empirische Pädagogik.

Wehner, K. (2016). Der Beginn: Gleichaltrigenbeziehungen im Kindergarten. In S.-M. Köhler, H.-H. Krüger \& N. Pfaff (Hrsg.), Handbuch Peerforschung (S.397 - 412). Opladen: Barbara Budrich.

Witton, T., Gramelt, K., Skalska, A. \& Thole, W. (2020). Kinder als ,Stakeholder' in Kindertageseinrichtungen. Soziale Passagen Journal für Empirie und Theorie Sozialer Arbeit, 11(1), 197 - 202.

Youniss, J. (1982). Die Entwicklung und Funktion von Freundschaftsbeziehungen. In W. Edelstein \& M. Keller (Hrsg.), Perspektivität und Interpretation. Beiträge zur Entwicklung des sozialen Verstehens (S.78-109). Frankfurt a. M.: Suhrkamp.

Youniss, J. (1994). Soziale Konstruktionen und psychische Entwicklung. Frankfurt a. M.: Suhrkamp.
Zinnecker, J. (2001). Stadtkids. Kinderleben zwischen Straße und Schule. Weinheim: Juventa.

\section{Autorenschaften}

Die Mitautor_innen sind erreichbar unter: Werner Thole, wthole@ uni-kassel.de; Barbara Lochner, barbara.lochner@fh-erfurt.de

\section{Förderung}

Open Access-Veröffentlichung ermöglicht durch Universität Kassel.

\section{Stephanie Simon}

Institut für Sozialwesen

Universität Kassel

Arnold-Bode-Straße 10

34127 Kassel

Simon.s@uni-kassel.de 